\title{
Plastic reconstruction and skin cover after injuries and burns
}

\author{
C. R. McLaughlin \\ M.A., M.B., F.R.C.S.E. \\ Consultant Plastic Surgeon, \\ Queen Victoria Hospital, East Grinstead
}

The PURPOSE of this paper is to summarize, for the benefit of busy surgeons in Casualty and Accident Departments, the role of plastic surgery in relation to injuries-including burns-in which the patient has suffered a serious breach of the 'skin envelope'; and also to consider certain aspects of facial injuries.

It is not easy to cover so wide a subject in a small space, and technical details can only be given for some of those procedures which lie reasonably within the compass of an Accident Department; but at the same time it is possible to hint at more complex procedures which may be carried out in an appropriate specialist unit.

The normal physiology of the body demands integrity of the skin envelope, and any serious breach in this may endanger life as well as health. In all serious burns and in many other injuries such a breach occurs, and the patient with a large exposed area suffers a double danger: on a systemic scale there is the hazard of a generalized infection where the entry of organisms can cause bacteraemia or septicaemia; and at the same time, particularly in burns, there is a gross disturbance of the body's biochemical balance. The local effects, also, are often serious; these include infection, oedema and pain. They do not necessarily endanger the patient's life but they are certainly a menace to comfort and often to function.

\section{Burns}

It will not be possible within the compass of this paper to consider the general management of the burned patient; nevertheless it is important to note that skin cover is extremely relevant to the survival of any patient with extensive burns; and until the breach in the skin envelope is repaired, his life may be in danger. We must now consider how best to repair this breach. The ideal procedure of course would be early primary grafting, but this is only possible where the burn itself can be excised within the first 4 or $5 \mathrm{hr}$. Primary excision is possible in only a surprisingly limited number of burns where the extent is small and the limits well-defined. One should beware of attempting primary excision where it is hard to assess the depth of the burn; this is notably true of the flexor aspect of the palm and fingers, particularly with electrical burns where depth is often impossible to assess precisely. However, given a small self-limited burn, such as may be caused by contact with hot metal, early excision and grafting as a single procedure within the first few hours is feasible and should not be overlooked.

\section{Delayed grafting}

In a vast majority of cases, early grafting being impossible, the surgeon must wait until nature, aided by later surgery, has established a raw area which will receive a graft. Sloughs of burned skin tend to be slow to separate and seldom does natural separation take place within the first 21 days. In unfavourable cases this period will be very much longer, and commonly in electrical burns it takes 5 or 6 weeks for separation to occur. It is also noteworthy that when burns have been treated by open exposure (and this is an excellent method in wellchosen cases) the dry eschar which has formed is much slower to separate than the moist slough of the covered burn. Thus it is clear that the timing both of spontaneous slough separation and of the surgical procedure of desloughing is important. The extensive removal of large sloughs is a major procedure even if the operation seems simple. It is essential that the general fitness of the patient should be assured and this usually involves a blood transfusion, if not before, at least during a procedure which can otherwise lead to dangerous exsanguination. For this and other reasons, speed during the operation is also imperative, as the duration bears some relation to the blood loss.

Much has been written of the advantages of chemical agents for desloughing, but these have been found on the whole to be disappointing, and when effective are extremely uncomfortable for the patient, and thus are badly tolerated.

It is most important to have clear criteria of what constitutes 'clean' granulations and emphasis has 
often been placed on precise bacteriology. This is not the whole answer and indeed culture reports should be used mainly to confirm clinical judgement and not to take its place. We must now consider methods of making the patient fit for surgery. Just as blood transfusion is important in the early resuscitation of the badly burned patient, so it must not be overlooked 3 or 4 weeks later when desloughing is contemplated. It is easy to have been misled by the early haematocrit values and to forget that the total mass of circulating red cells is invariably reduced by a severe burn; this latent anaemia declares itself increasingly after the first week. During these early weeks the patient should be on a high protein diet, but there is little to be gained by special drugs such as cortisone.

The second part of the problem is to prepare the raw area locally so that it is sufficiently clean for a Thiersch graft to be applied with a good expectation of a successful 'take'. This problem has received much attention, but a good deal of confusion still exists in the minds of young doctors. Many centres for the treatment of burns rely on the use of prophylactic systemic antibiotics given over a longish period and this cannot be readily condemned, though its advantages are a little uncertain. There is certainly a place for the use of topical antibiotics where a particular organism shows signs of being troublesome, and certain organisms (notably $P s$. pyocyanea) are notorious for causing the failure of grafts. In some cases an antibiotic solution made up in suitable strength (such as chloramphenicol, 1 part in $\mathbf{4 0 0}$ in normal saline) can be applied as an old-fashioned cold compress on gauze, the latter being covered with sterile jaconet to keep the dressing moist. However, this method should only be used for a limited time to avoid colonization of the granulations by resistant organisms. The use of so-called local antiseptics is of very doubtful value though there has been a considerable vogue for such substances. However, we can reasonably say that those antiseptics which kill organisms are very apt to kill the tissues, or at least to cause the patients a degree of pain which they will not readily tolerate. In many cases straightforward attention to simple surgical hygiene, the use of a bath containing hypochlorite (or Dettol), and the application of a bland dressing are the first requirements. Everyone is anxious to select a dressing which does not adhere and so does not cause pain and bleeding when it is changed, and tulle gras in its various forms has had a great vogue. While there is no objection to this material during the first week or two, it is apt in the later stages to cause progressive maceration of the wound. As it happens, ordinary gauze is probably the best material to use next to a wound that has become a little indolent. The choice of bland medications to apply on the gauze is very wide.
For many years plastic surgeons have favoured a mixture of eusol and liquid paraffin in equal quantities. These two substances are, of course, not miscible, but pharmacists can produce an emulsion. In either form this combination is effective, since the paraffin is satisfactorily bland and the eusol (which is, of course, sodium hypochlorite) is reasonably effective as a hypertonic solution which encourages a healthy exudate, and is one of the few acceptable local antiseptics. In terms of simple hygiene the use of a bath is to be looked on with considerable favour, and the theoretical objection of faecal and other contamination is usually outweighed by the advantages of simple mechanical lavage.

\section{The scope of surgical desloughing}

In excising sloughs it is important to reach tissues which are manifestly viable; with fat this is difficult to assess, though in children the fat is notably more vascular than in adults. It is usually wise in deep burns of the abdomen and thighs to carry the desloughing down to the deep fascia, as this is often the first layer that one can be sure is safely viable.

As regards the type of graft to be used, split skin from the patient himself is, of course, the first choice. Homografts whether taken from relatives or a cadaver will in few circumstances last longer than 3 weeks, and their use is thus distinctly limited. It is, however, possible to store the patient's own autografts taken at one operation so that they can be applied, if necessary without an anaesthetic, at a rather later stage when the condition of the raw areas is more satisfactory. This applies when one part of the burn is surgically more advanced than another. In the extensively burned patient, proper priority must be given to those areas which merit it; attention to the face, and above all to the eyelids, must come first. The hands are of special importance, and great attention must also be paid to those parts of the limbs which undergo flexion.

\section{Technique of cutting skin grafts}

There can be no excuse these days for a young surgeon being unable to cut a split skin graft of reasonable size. This is essentially a simple procedure and just as much part of the emergency surgeon's repertoire as passing a catheter.

Only two pieces of equipment are required; the first is one of the modern skin grafting knives, with an adjustable guard to control the thickness of the graft. Perhaps the ideal design (Cobbett, 1967, personal communication) is a knife with a guard which oscillates but does not rotate (since rotation tends to roll a very thin graft up like a blind, which can be awkward) (Fig. 1). The other requirement is a bevelled wooden board to hold the skin taut and flat in front of the knife. The best areas for 


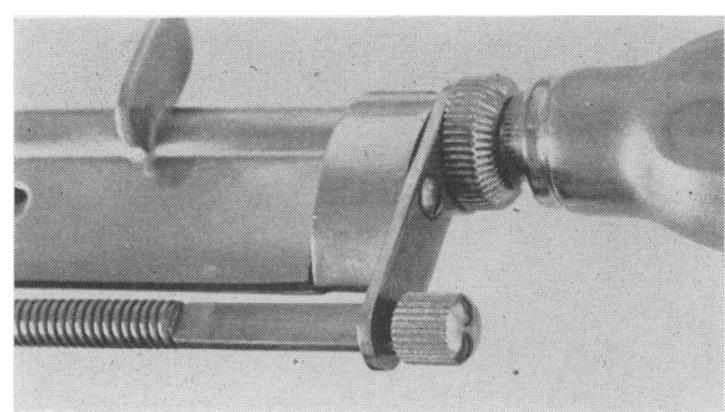

FIG. 1. Skin grafting knife with mobile guard. The guard does not rotate but stays pressed steadily on the skin while the knife glides to and fro.

cutting grafts are in the inner aspect of the thigh and upper arm, and the assistant should so hold the limb as to present a flat surface of maximum width (Fig. 2). The most useful graft is roughly half the

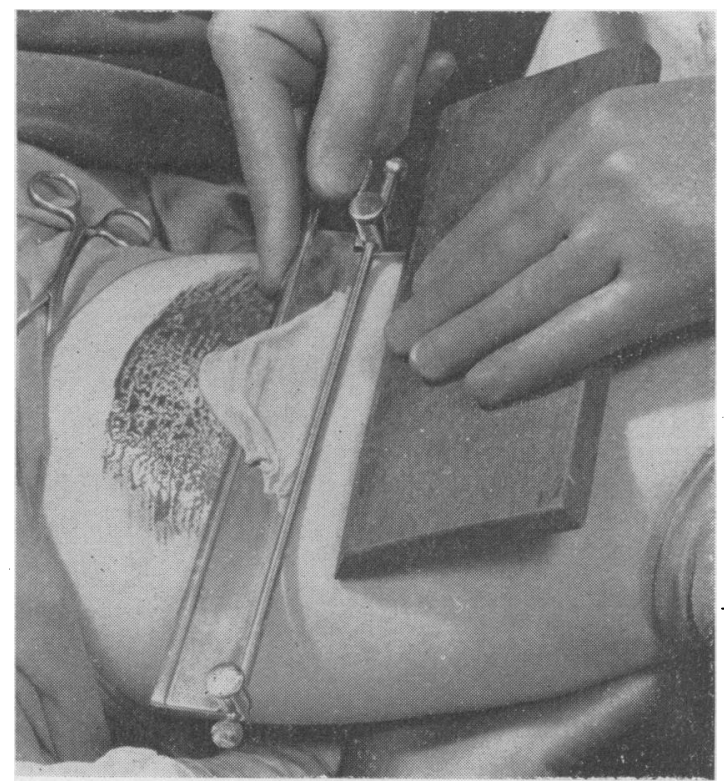

FIG. 2. The patient's thigh presented flat, supported by the assistant's hand below it. In favourable cases this graft may be 3 or 4 in. wide and extend the full length of the thigh.

full thickness of the skin approximately $15 / 1000 \mathrm{in}$.) The cut skin is spread on tulle gras at full stretch, and the donor surface is itself dressed with tulle gras.

In any badly burned patient the surgeon will have considerable difficulty in finding sufficient donor areas to obtain the skin he wants, but if the skin is taken with skill it is possible for any one area to provide a second graft quite soon. It may surprise some readers that in specially favourable cases three lots of thin skin have in fact been cut within a total of 3 weeks from the same area. If homografts are being used it is important that they should be laid on alternately with the autografts, so that the latter can spread and replace the homografts as these are progressively rejected. There is a good review of these problems by Wallace (1966).

\section{Degloving injuries}

Patients who are injured in road traffic or industrial accidents frequently suffer what is conveniently called 'degloving' of upper or lower limb. Here there is sudden loss of skin as in a burn, but the problem is simpler and the systemic effects are usually less severe. A good deal of judgement is required in deciding whether to return the flap of skin which has been stripped back; this obviously should not be done if the skin is manifestly dead. However, it is sometimes possible by severe trimming to retain the proximal part of the traumatic skin flap and use it to cover part of the exposed tissues. Where skin is totally detached it can, in rare cases, be filleted and returned as a full-thickness Wolfe graft. This does not have many applications outside the scalp.

\section{Compound fractures of the leg}

The problem of treating limb fractures which are not only compound but are associated with an actual loss of adjacent skin is a complex and fascinating one. There is a good review (Brown, 1965) which deals with the lower limb and discusses the alternatives. In these cases the scope of debridement must be correctly assessed. The only simple generalization is that all viable tissue must be retained, and tissues which are almost certainly dead must be sacrificed. If muscle does not bleed it should usually not be retained, and bony fragments when loose and detached from periosteum are seldom worth keeping, being in fact very prone to end up as sequestra. If the area of skin which has been lost is small then it may be possible to close the wound by primary suture. This, however, must not in any circumstances be done under tension; also every precaution must be taken against local haematoma formation and local sepsis. Any of these will lead to disruption of the wound, and the position then will be worse than if primary suture had not been attempted. In difficult conditions it is best simply to apply a Thiersch graft to the soft tissues. Elaborate repairs using flaps, whether local or from the opposite leg, are very risky and can seldom be justified. It has been fashionable to carry out so-called 'relaxation incisions' in a somewhat crude form to allow primary closure, but again this is seldom justified, and grafting of the secondary raw area is essential. In some cases the best alternative is delayed primary suture at about 6 days. 
If the local conditions are extremely unpromising, then delayed suture must be discarded and the patient treated by the secondary application of skin grafts to the tissues when they develop healthy granulations. Grafts can, of course, take on periosteum and they will also take on cancellous bone. The one surface that is hopeless to graft is the bare cortex. Provided the fracture is properly immobilized it is surprising how often the wound can in fact be re-surfaced entirely by Thiersch grafts. Even if this is only a provisional cover, it is nevertheless well worth carrying out, and the whole problem of secondary cover can be left to experts at a later stage.

\section{Facial injuries}

In relation to facial injuries there are some special factors, and these may be summarized as follows:

(1) Any residual scarring is likely to be offensive, at least to the patient.

(2) The blood supply of the face is for the most part superb, and wide excisions are seldom necessary.

(3) There is an absolute obligation to curette tar-ingrained surfaces whether abrasions or lacerations. If this is not done the patient will have a permanently pigmented scar and may well guess that this was due to the neglect of the original surgeon!

(4) As regards facial fractures, those involving the nasal bones are the most likely to be compound.

(5) A closed fracture in the orbital area may seriously involve the dura, the eye or its related muscles. (Fractures of maxilla and mandible will not be considered here as they lie outside the scope of this paper.)

We may now examine these local factors more closely. It is often impossible to get a good cosmetic result when repairing a facial laceration at the time of injury. It is, of course, obligatory to carry out the repair meticulously as though it were in fact the final operation; but a disappointing result does not necessarily reflect on the surgeon. Gentle handling, using fine instruments and sutures, is imperative. Hypertrophy of the scar may be aggravated by small retained fragments of foreign material, and these are very easily missed. In cases of true (or apparent) keloid formation it is wise to wait up to 6 months before carrying out a secondary excision and suture. Owing to the excellent viability of the facial skin, it is usually sufficient to remove a mere millimetre or two of the skin margins prior to primary suture, unless the edges are torn in an irregular way.

A casualty surgeon must always assume that a patient who has been injured by contact with a modern road has in fact got tar tattooed into his wounds. This is hard to detect at the time where the tar is concealed by blood, but it is absolutely

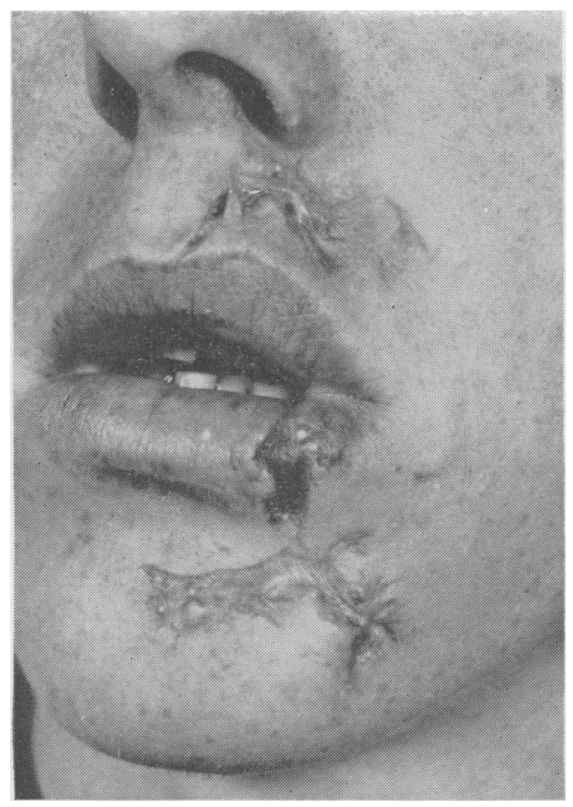

FIG. 3. Patient with heavily tar-ingrained scars shown following primary surgery, which clearly did not include adequate curetting.

essential to treat it properly, as there is often ne chance of recovering later the opportunity initiall missed (Fig. 3). Apart from soap and water and scrubbing brush, the best instrument is a steel spoon such as Volkmann's and this must be plied ruthlessly even though the process appears to be traumatic. Each raw abraded surface and each deep fissure must be curetted in turn, so that there is as little chance as possible of these ugly tattoos appearing later.

Turning to nasal fractures, simple early reduction is important and for this manipulation the best instrument is Walsham's. It may be necessary after correction of the fracture to pack the nose, and in rare cases to insert short rubber tubes to maintain an otherwise blocked airway. Surgery to the septum, in any definitive sense, it not possible until months later. Splinting of a badly fractured nose may appear difficult, and there are two methods available. It is usually sufficient to apply plaster of Paris in $\frac{D}{O}$ the form of a triangle with an extension on to the forehead. The size of this triangle can be roughly o estimated by first cutting a piece of lint to fit the nose (Fig. 4). This method has the advantage that the fracture can be finally manipulated through the plaster as it dries. In very bad cases where the nasal bones have collapsed, the proper procedure is to cut out two lead plates about $1 \times \frac{1}{2}$ in. which will lie on each side of the fracture. Their upper ends must reach above the level of the inner canthus. Two holes are punched in the lead plates which are 


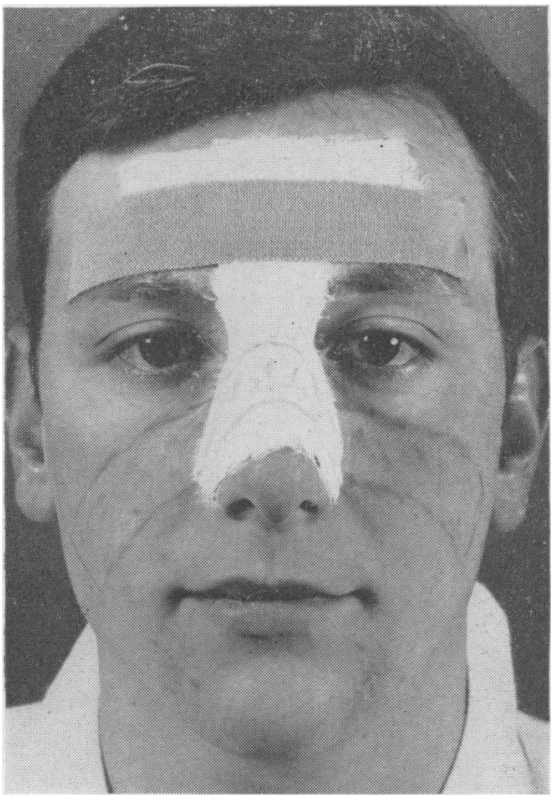

FIG. 4. Plaster of Paris nasal splint in position. The lower piece of strapping at cheek level has been omitted to show the design of the plaster.

then transfixed by a wire suture mounted on a sharp needle. This is passed (say) from left to right through the upper holes and back through the lower ones, in the form of a mattress suture. When tied firmly but not tightly, this wire will maintain the bony fragments in a reasonably accurate position and can be left for a week.

Note: As regards fractures around the eye, it is important if possible to obtain the advice of an ophthalmic colleague. It must always be remembered that the injury which fractured the orbital ring may have caused serious damage to the globe or to the optic nerve, and it is usually not difficult to make a rough assessment of vision prior to surgery.

A suspected fracture of the malar bone should be confirmed by X-ray. It is important, unless chemosis makes this impossible, to test the patient for diplopia by asking him to follow one's finger and say if he has double vision. The fractured malar should be reduced by one of the recognized routes (either the upper temporal route, through the temporal fascia, or, in selected cases where the antrum is collapsed, by a Caldwell-Luc approach). If the fracture remains unstable, and a gap still be felt in the region of the fronto-malar suture, then open wiring at this point may be indicated. Happily a proper reduction of the malar or orbital fracture along these lines is usually, but not invariably, sufficient to cure the diplopia. If it remains then it must be seen by a specialist as early as possible.

Note: In connection with severe nasal fractures we may consider for a moment the problem of cerebro-spinal rhinorrhoea. This is usually an indication that there is a maxillary fracture in addition to a nasal fracture, and such cases should be treated by experts. It is, however, useful to recall that primary treatment by accurate reduction and immobilization almost invariably cures the leak of CSF within a matter of hours, and the intervention of a neurosurgeon is seldom called for. With proper surgery plus antibiotics the incidence of meningitis is virtually nil in this context.

\section{Conclusions}

It can be seen from this necessarily brief summary that reconstruction and skin cover in relation to burns and injuries presents many problems. Some of these patients should clearly be dealt with by experts, but such experts are not always immediately available, even these days. The main purpose of these clinical suggestions, necessarily made in a somewhat didactic form, is that the surgeon on the spot should do nothing and omit nothing which will complicate the later treatment. A mistake in the primary treatment, such as failing to curette a taringrained face, or perhaps closing a compound fracture under tension so that the whole wound breaks down, may make the subsequent treatment not merely difficult but impossible.

\section{Acknowledgment}

I am grateful to Mr P. Broadbery, A.R.P.S., A.I.I.P., for the photographs.

\section{References}

Brown, R.F. (1965) The management of traumatic tissue loss in the lower limb, especially when complicated by skeletal injury. Brit. J. plast. Surg. 18, 26.

WALlaCe, A.F. (1966) The problem of skin cover in extensive burns. Brit. J. plast. Surg. 19, 161. 